\title{
Unique Presentation of Cerebellopontine Angle Choroid Plexus Papillomas: Case Report and Review of the Literature
}

\author{
Mark Anderson ${ }^{1}$ Parker Babington ${ }^{2}$ Reza Taheri ${ }^{3}$ Mairo Diolombi ${ }^{4}$ Jonathan H. Sherman ${ }^{2}$
}

${ }^{1}$ School of Medicine, The George Washington University School of Medicine, Washington, District of Columbia, United States

2 Department of Neurosurgery, The George Washington University, Washington, District of Columbia, United States

${ }^{3}$ Department of Radiology, The George Washington University, Washington, Washington, District of Columbia, United States

${ }^{4}$ Department of Pathology, The George Washington University, Washington, District of Columbia, United States

\author{
Address for correspondence Jonathan $\mathrm{H}$. Sherman, MD, 2150 \\ Pennsylvania Avenue, NW Suite 7-420, Washington, DC 20037, United \\ States (e-mail: jsherman0620@gmail.com).
}

J Neurol Surg Rep 2014;75:e27-e32.

\begin{abstract}
Objectives We present the case of a choroid plexus papilloma (CPP) in the cerebellopontine angle (CPA), describe the different appearances of CPPs with a variety of imaging techniques, and discuss the differential diagnosis of CPA tumors.

Participant and Design We report the case of a 52-year-old woman with headache, tinnitus, and unilateral hearing impairment whose preoperative magnetic resonance imaging revealed a heterogeneously enhancing CPA mass that extended into the internal auditory canal.

Main Outcome Measures, Results, and Conclusion The preoperative imaging appearance of the lesion was most consistent with that of a schwannoma. Postoperative histopathologic examination found the tumor to be a CPP with cuboidal epithelial cells

Keywords

- cerebellopontine angle

- choroid plexus papilloma overlying fibrovascular stroma. CPPs are rare benign central nervous system neoplasms arising from choroid plexus epithelium. The most common site of presentation is in the fourth ventricle in adults and the lateral ventricles in children. CPPs rarely occur in the CPA, and when they do, clinical-radiologic diagnosis is difficult due to both the rarity of this presentation and to nonspecific radiological features.
\end{abstract}

\section{Introduction}

Choroid plexus papillomas (CPPs) are rare benign intracranial tumors arising from the cuboidal epithelial lining of the choroid plexuses. They comprise $<1 \%$ of all intracranial tumors and more commonly occur in childhood, although they are reported in adulthood as well. ${ }^{1-3}$ CPPs arise where choroid tissue is present, most commonly in the lateral ventricles in children and in the fourth ventricle in adults. ${ }^{4}$ Although benign, CPPs may metastasize, mandating total surgical resection if possible. ${ }^{5,6}$ Rarely, CPPs can present as

received

October 27, 2012

accepted after revision

September 1, 2013

published online

December 12, 2013 cerebellopontine angle (CPA) tumors from direct extension of the tumor out the foramen of Luschka or from seeding along cerebrospinal fluid pathways. ${ }^{7-10}$ When a CPP presents as a CPA lesion, clinical diagnosis is not straightforward because symptoms from tumors in this area correlate more with which nerves and cerebral structures are involved, rather than the specific tumor type. ${ }^{11}$ Radiographic appearance of this lesion can overlap with the more common CPA lesions including acoustic neuromas and meningiomas. ${ }^{12-15}$ We report the case of a patient whose preoperative imaging highlighted this potential overlap.
License terms

Stuttgart · New York

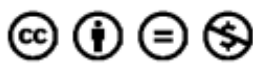




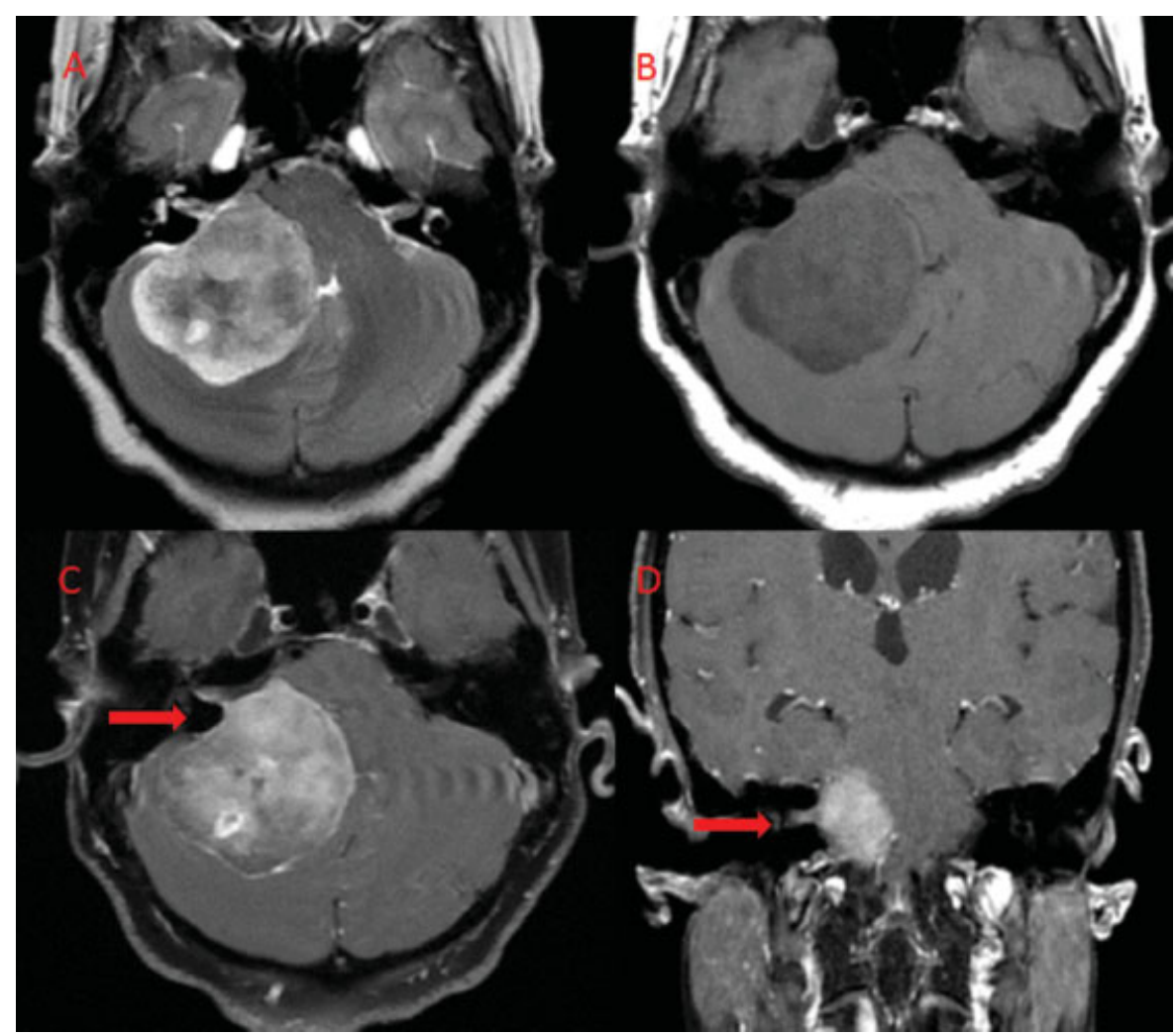

Fig. 1 Preoperative magnetic resonance imaging (MRI). (A) Axial T2-weighted MR image displaying a well-circumscribed heterogeneous extraaxial mass centered in the right cerebellopontine angle (CPA). The lesion is causing marked mass effect on the right middle cerebellar peduncle (right brachium pontis) and the fourth ventricle resulting in mild hydrocephalus (not shown). (B) Axial T1-weighted MR image displaying the right CPA mass to be hypointense with respect to brain parenchyma. (C) Axial T1-weighted contrast-enhanced MR image displaying heterogeneous enhancement of the right CPA mass. In addition, there is evidence of contrast enhancement extending through the porous acusticus and along the posterior wall of the left internal auditory canal (arrow). (D) Coronal T1-weighted contrast-enhanced MR image identifying the extension of contrast into the internal auditory canal (arrow).

\section{Case Report}

A 52-year-old woman presented to the neurosurgery clinic with a history of headaches since a ground-level fall 1 year prior. The headaches were progressively worsening and were associated with difficulty hearing and tinnitus in the right ear. The patient's past medical history was otherwise noncontributory. On physical examination the patient was awake and alert with normal orientation and cognition. Pupils were equal and reactive with full visual fields. There was slight right facial weakness at the orbicularis oris muscle. Hearing was decreased to gross testing on the right. Motor examination was full strength throughout. Sensation was intact to light touch and pinprick.

Magnetic resonance imaging (MRI) of the brain revealed a well-defined, heterogeneous $4.4 \times 4.2 \times 3.7 \mathrm{~cm}$ extra-axial CPA mass that was predominantly hypointense on T1 and hyperintense on $\mathrm{T} 2$ with respect to the brain parenchyma. This mass was compressing the fourth ventricle and right cerebellar peduncle causing mild hydrocephalus. After the administration of contrast, the lesion displayed moderate heterogeneous enhancement. Contrast enhancement was seen extending along the posterior aspect of the internal auditory canal (-Fig. 1). The patient underwent a cerebral angiogram that displayed a relatively hypovascular mass in the right CPA. The right anterior inferior cerebellar artery (AICA) was mildly displaced and was smaller in caliber as compared with the left AICA. The right posterior inferior cerebellar artery (PICA) was grossly unremarkable (-Fig. 2). Based on the imaging

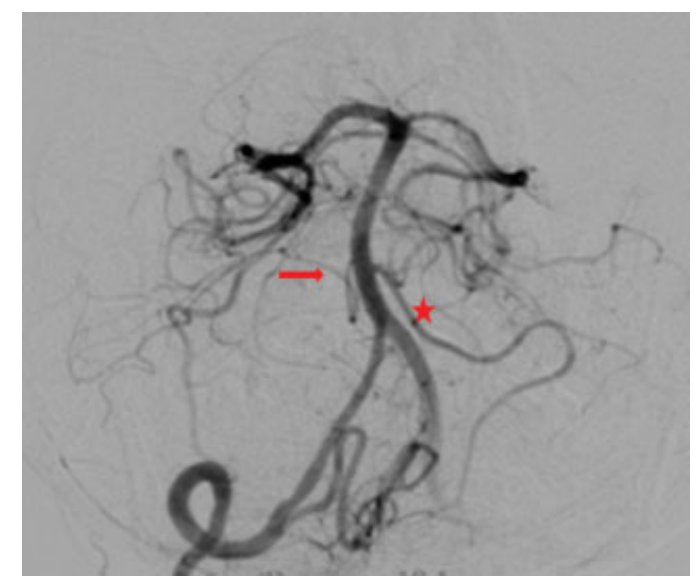

Fig. 2 Cerebral angiogram. An exaggerated transfacial view of a selective right vertebral artery injection did not show a clear area of enhancement in the right cerebellopontine angle. The caliber of the mildly displaced left anterior internal cerebellar artery (arrow) is smaller than the right side (star). 


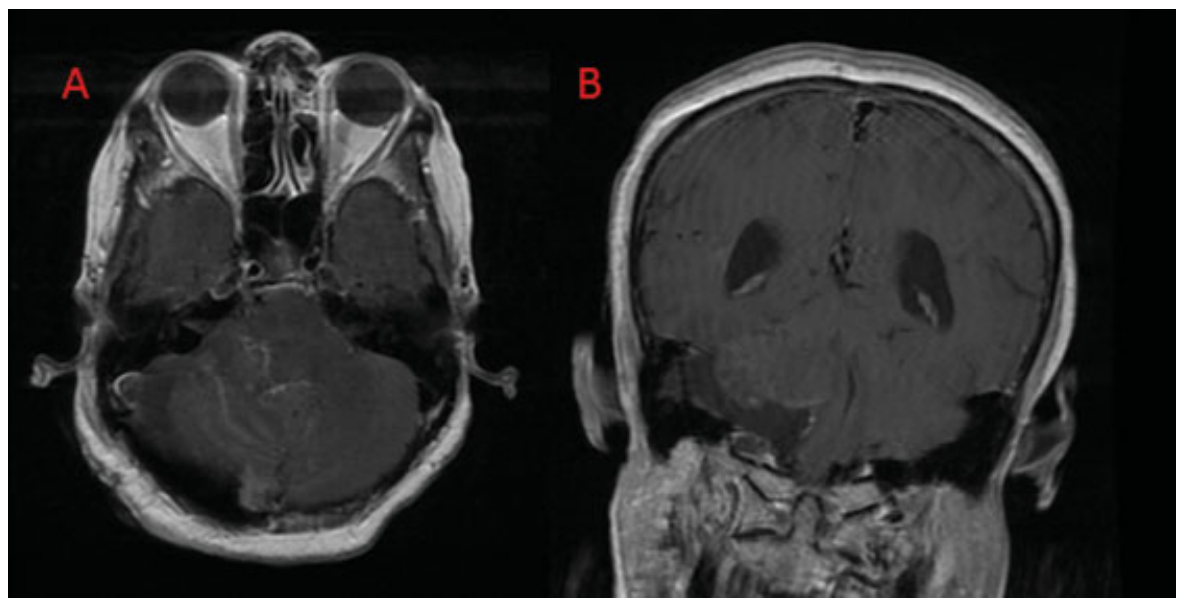

Fig. 3 Postoperative magnetic resonance (MR) imaging. (A) Axial T1-weighted contrast-enhanced MR image displaying no residual contrast enhancement. (B) Coronal T1-weighted contrast-enhanced MR image displaying no residual contrast enhancement.

appearance of this mass, it was presumed that the lesion likely represented a schwannoma.

The patient was subsequently taken to the operating room and underwent a right suboccipital craniotomy via an extended retrosigmoid approach and resection of the CPA lesion (-Fig. 3). A large, soft, and vascular tumor with compression of the cerebellum medially and superiorly was resected except for a $2 \times 2 \mathrm{~mm}$ piece that was left on the root entry zone of the right facial nerve. The tumor was densely adherent to the dura adjacent to the internal auditory canal (IAC) where it had parasitized the dural blood supply. However, there was a nice arachnoidal plane between the tumor and the cranial nerve (CN) VII and VIII complex, and the tumor was found not actually to enter into the IAC. The only location that did not have a nice plane was located at the CN VII dorsal root entry zone where a 2-mm portion of tumor was coagulated but not fully resected. Branches of vertebral artery, PICA, and AICA were identified and preserved. Lower CNs adherent anterior to the tumor were identified, dissected free of the mass, and preserved. Following significant tumor debulking, the mass was found to be attached to choroid plexus upon its exit from the foramen of Luschka. At intraoperative

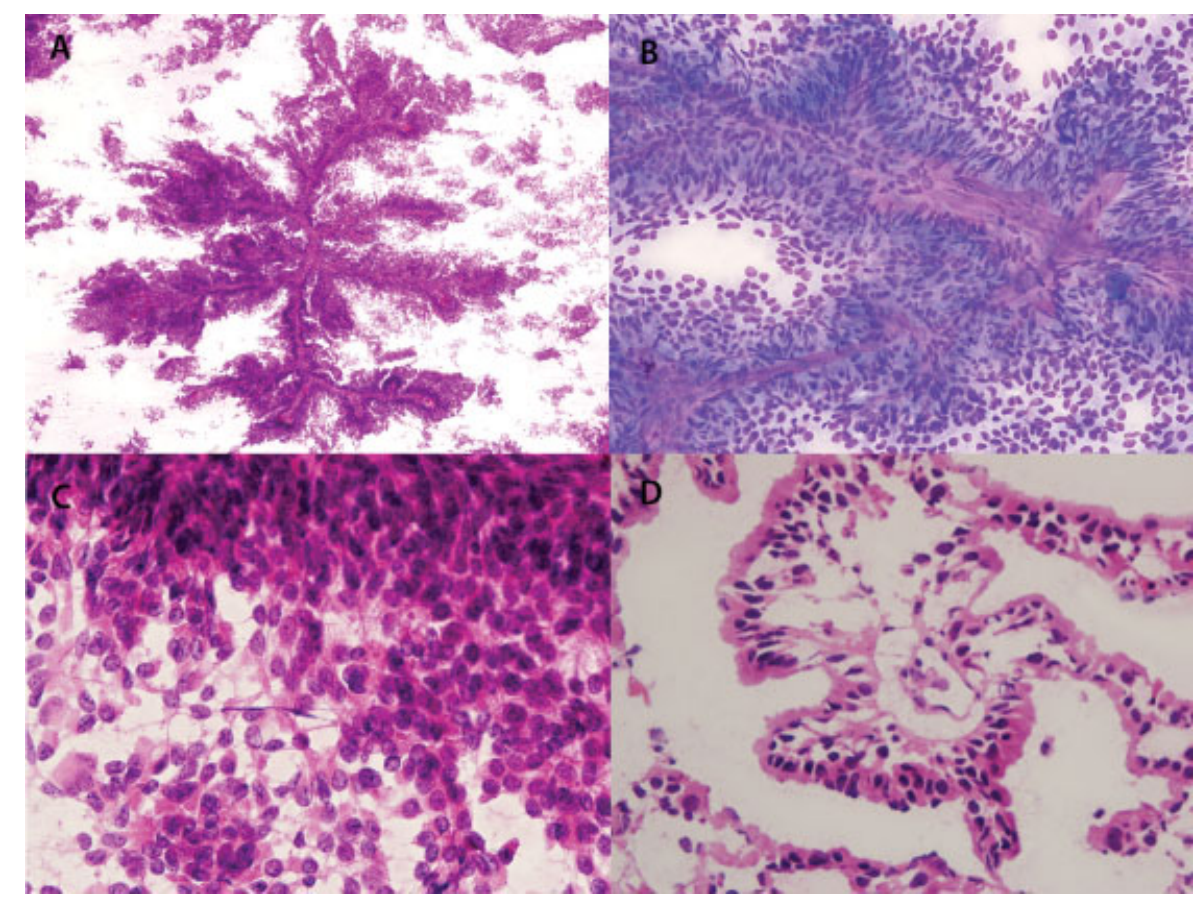

Fig. 4 (A) Hematoxylin and eosin (H\&E) stain $(\times 40)$ showing a hypercellular smear with a papillary fragment of tissue. (B) Romanowsky stain $(\times$ 200) showing a fibrovascular structure lined by bland, crowded, and pseudostratified epithelial cells. (C) H\&E stain $(\times 400)$ showing thin cytoplasmic processes coming off bland-appearing epithelial cells. (D) Frozen section H\&E $(\times 400)$ showing bland epithelial cells lining a fibrovascular core with distinct cytoplasmic borders. Neuropil elaboration is not identified. 
consultation a small biopsy of the lesion showed a cauliflower-like macroscopic appearance and yielded hypercellular Diff-Quik and hematoxylin and eosin (H\&E) smears. Microscopic study of the smears disclosed abundant papillary tissue fragments with bland cells arranged along vesselcentered stalks. Some of the cells were surrounded by frayed cytoplasm suggesting possible neuropil elaboration, but a subsequent cryostat section disclosed that most cells possessed clearly defined cytoplasmic margins without process formation (-Fig. 4). Formalin-fixed paraffin-embedded tissue sections stained with $\mathrm{H} \& \mathrm{E}$ disclosed a papillary neoplasm without neurophil. Pseudostratified epithelial cells were seen lining the fibrovascular stalks with monotonous and eccentrically located nuclei. There was no evidence of necrosis, neural tissue invasion, or mitosis. Immunohistochemical studies for Ki-67, glial fibrillary acidic protein, and CAM 5.2 were performed at our institution and showed a strong and diffuse cytoplasmic staining pattern for the latter two and a low proliferation index ( $2 \%$ qualitative estimate). A transthyretin (TTR; prealbumin) study performed and interpreted at PhenoPath Laboratories showed strong patchy cytoplasmic positivity. The microscopic findings and immunophenotype supported the diagnosis of a choroid plexus papilloma (-Fig. 5). One month postoperatively the patient had intact $\mathrm{CN}$ function with resolution of facial asymmetry and subjective improvement of hearing.

\section{Discussion}

Primary CPPs usually develop within the ventricular system; most tumors are located in the fourth and lateral ventricles.
Some of these lesions may extend from the ventricular system to extraventricular regions, but only rarely have CPPs been reported to be located primarily in extraventricular regions. Most primary extraventricular CPPs have been reported in the CPA. ${ }^{16,17}$ CPPs are generally solitary tumors, although multifocal presentations have been described. ${ }^{12,18,19}$ Surgical resection is the treatment of choice for CPPs with radiation treatment reserved for recurrent disease. ${ }^{1,18,20}$ Because the intraoperative pathology was not consistent with a highgrade lesion, leaving a small amount of tumor adherent to the facial nerve was believed to be in the patient's best interest keeping in mind that 5-year survival rates for CPP have been found to be $100 \%$ with gross total resection and $94 \%$ for subtotal resection, whereas a facial nerve palsy can be a significant morbidity. ${ }^{37}$

CPA lesions are clinically nonspecific, and the presenting symptoms are related more to the nerves or cerebral structures involved with the lesion than the nature of the lesion itself. Dysfunction of hearing and facial movement, headache, dizziness, papilledema, ataxia, and hydrocephalus are common presentations of CPA CPPs, although other findings have been reported including hypoglossal neuropathy and trigeminal neuralgia. $7,8,17,18,21-25$ Our patient presented with a group of nonspecific clinical signs and symptoms typical of CPA lesions that included headaches, unilateral hearing loss, tinnitus, hydrocephalus, and right facial weakness. There is no single pattern of clinical manifestations of CPA CPPs such that these lesions mimic other tumors common to the CPA while others may imitate cerebellar or jugular foramen lesions. More common lesions that can present with similar clinical manifestations make up a significantly larger

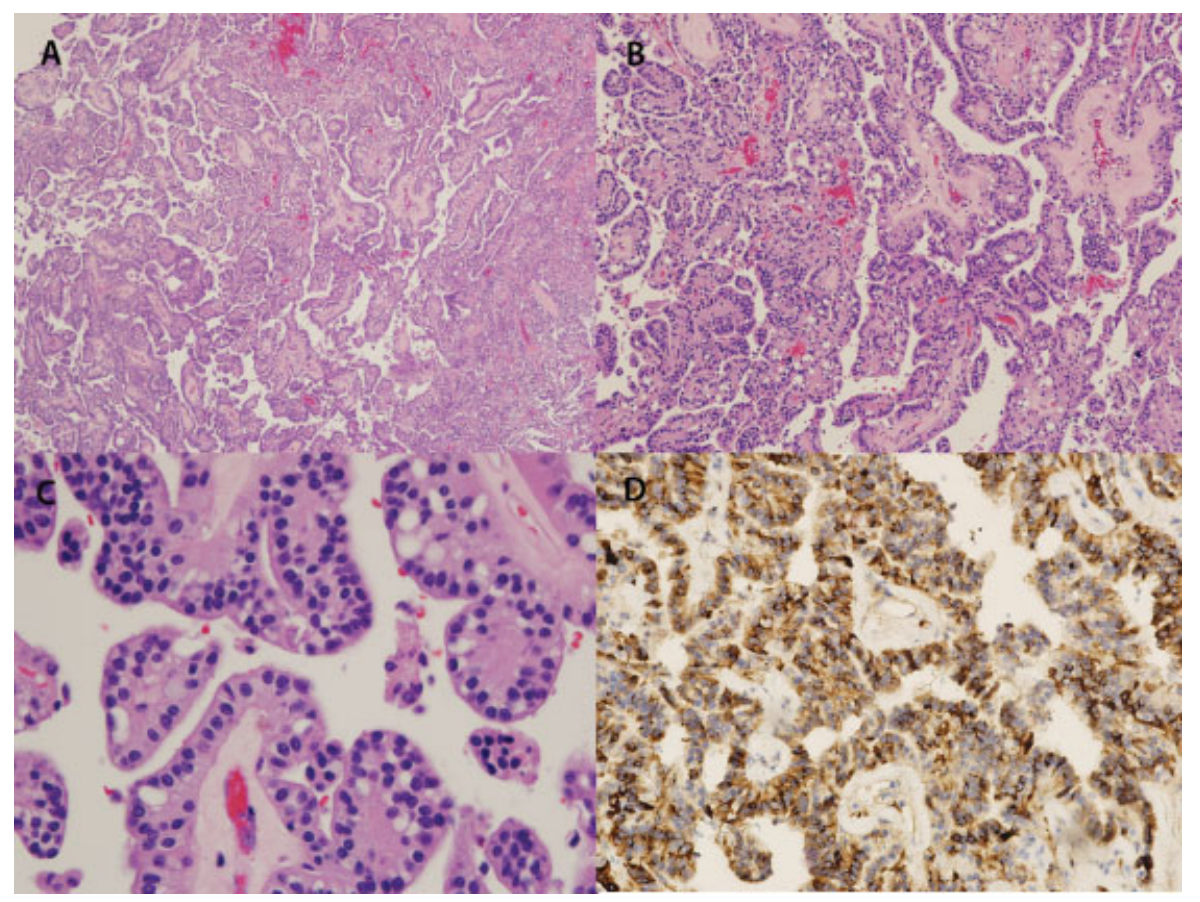

Fig. 5 (A) Permanent section hematoxylin and eosin (H\&E) $(\times 40)$ showing fibrovascular cores lined by bland epithelial cells. (B) Permanent section H\&E ( $\times 100)$ (higher magnification of Fig. 2A). (C) Permanent section H\&E $(\times 400)$ (higher magnification of Fig. 2B) showing crowded columnar cells with a high nuclear to cytoplasmic ratio. No atypia, necrosis, or significant mitotic activity is identified. (D) Transthyretin antibody (×200) highlighting the epithelial cells. 
percentages of CPA tumors, including vestibular schwannomas (70-80\%), meningiomas (10-15\%), and epidermoid cysts (5\%). ${ }^{11}$ The remaining CPA tumor types, including CPPs, make up $<1 \%$ each of all CPA tumors, and consequently they are challenging to diagnose. Because the clinical presentations of CPA CPPs are so varied, imaging studies are the primary means for preoperative diagnosis.

On noncontrast head computed tomography (CT), CPPs are isodense to hyperdense as compared with brain parenchyma. Internal calcification is present in up to $20 \%$ of cases. ${ }^{6,13-15,17,22}$ On MRI, CPPs are typically isointense to hyperintense on T2 and isointense to hypointense on T1. Most CPPs show robust and homogeneous enhancement on both CT and MR images. ${ }^{13-16,25-28}$ In rare cases, the vascular pedicle can twist, leading to infarct of the tumor and dense calcification. Heterogeneous enhancement as seen in this case is uncommon. ${ }^{15}$

CPPs are typically quite vascular and display intense tumor blush on catheter-guided cerebral angiography. ${ }^{6}$ On cerebral angiogram, prolonged contrast enhancement, arteriovenous shunting, and enlargement of the feeding artery, AICA in most cases, are typical features of CPA CPPs. ${ }^{12,16,25,29,30}$ Arteriographic studies of CPP vasculature have noted consistent AICA dilation with numerous and irregular feeding branches concentrated in the CPA or jugular foramen, encircling the CPPs. Zhang et al reviewed a series of 60 CPA tumor arteriograms that included acoustic neuromas, meningiomas, angioreticulomas, gliomas, tumors ${ }^{25}$ of the jugular foramen, chordomas of the clivus, and metastatic tumors and found that no other CPA tumor type shared the characteristic angiographic features of CPPs: AICA dilation with irregular dilated branches surrounding the tumor. Less commonly, PICA may be the primary arterial feeder of CPA CPPs. ${ }^{6,8,16,30,31}$ Rarely the tumor blush is less robust and nonspecific. ${ }^{9}$ The lack of a tumor blush with a small PICA as compared with the opposite side is quite uncommon for CPPs.

Schwannomas are typically isodense to the brain parenchyma on noncontrast CT. Unlike CPPs, internal calcifications are rare. On MRI, they can have a variable appearance based on the composition of Antoni type A and B cell types. These tumors are generally isointense to hypointense to the brain parenchyma on T1 and can have a heterogenous T2 signal hyperintensity. Although the cystic components of the tumor tend to be T2 hyperintense, foci of microhemorrhages appear as areas of T2 signal hypointensity. After the administration of contrast, these lesions can avidly enhance. On cerebral angiogram, schwannomas are typically hypovascular. The imaging findings of this patient are atypical for a CPP, but they are also to some extent atypical for a schwannoma. In - Fig. 1, T20weighted images appear to show the nerves of the IAC with enhancement that extends into the IAC exclusively along the posterior dural border, instead of circumferentially within the IAC, as would be more typical of a schwannoma. The enhancement into the IAC seen on brain MRI in our patient is likely secondary to a reaction to the tumor involvement at the dural edge adjacent to the IAC and not actually from tumor extension.

A recent literature review by Zimny et al suggested that although CPA meningioma, schwannoma, and CPP tumors may show a very similar appearance on conventional MR imaging, they may differ in perfusion-weighted imaging (PWI). ${ }^{27}$ Perfusion imaging can estimate the cerebral blood flow, cerebral blood volume (CBV), and mean transit time of a tumor or a region of the brain. A low CBV tumor was more likely to represent a schwannoma or CPP than a meningioma. The distinction between schwannoma and CPP was less apparent based on parameters measured based on PWI.

The role of MR spectroscopy (MRS), which provides information about the metabolic profile of a tumor, has also been studied in distinguishing between different CPA lesions. Among common CPA tumors, characteristic features include high alanine and low $\mathrm{N}$-acetylaspartate (NAA) peaks in meningiomas, high myoinositol in schwannomas, and the presence of lactate/lipid and choline peaks in metastases. ${ }^{11,32,33}$ CPPs show a characteristically high myoinositol level ( $>10 \mathrm{mmol} / \mathrm{kg}$ ) as well as a complete absence of creatine and NAA. ${ }^{34-36}$ Thus, although MRS may help in distinguishing schwannomas from meningiomas by demonstrating a prominent myoinositol peak in schwannomas versus alanine peak in meningiomas, like relative $\mathrm{CBV}$ this modality cannot readily differentiate CPA schwannomas from CPPs, both of which demonstrate a myoinositol peak.

In conclusion, we present a case of a 52-year-old woman who presented with nonspecific clinical symptoms characteristic of CPA tumors whose MRI and angiographic imaging studies showed a heterogeneous enhancement and lack of a robust tumor blush. As described, these features are most consistent with a schwannoma, especially in combination with enhancement within the IAC. There is considerable overlap between the perfusion and spectroscopic appearance of a schwannoma and CPP, making the impact of these modalities of questionable value. Choroid plexus papilloma should be considered in the differential diagnosis of CPA masses, even in the presence of a heterogeneously enhancing hypovascular lesion.

\section{References}

1 McGirr SJ, Ebersold MJ, Scheithauer BW, Quast LM, Shaw EG. Choroid plexus papillomas: long-term follow-up results in a surgically treated series. J Neurosurg 1988;69(6):843-849

2 Chari G, Dhaygude A, Kulkarni MV, Patkar SV, Kalgutkar A. Choroid plexus papilloma. Indian Pediatr 1994;31(10):1305-1308

3 Wolff JE, Sajedi M, Brant R, Coppes MJ, Egeler RM. Choroid plexus tumours. Br J Cancer 2002;87(10):1086-1091

4 Strojan P, Popović M, Surlan K, Jereb B. Choroid plexus tumors: a review of 28-year experience. Neoplasma 2004;51(4):306312

5 Enomoto H, Mizuno M, Katsumata T, Doi T. Intracranial metastasis of a choroid plexus papilloma originating in the cerebellopontine angle region: a case report. Surg Neurol 1991;36(1):54-58

6 Furuya K, Sasaki T, Saito N, Atsuchi M, Kirino T. Primary large choroid plexus papillomas in the cerebellopontine angle: radiological manifestations and surgical management. Acta Neurochir (Wien) 1995;135(3-4):144-149

7 Khoddami M, Gholampour Shahaboddini R. Choroid plexus papilloma of the cerebellopontine angle. Arch Iran Med 2010;13(6): 552-555 
8 Kumar R, Achari G, Benerji D, Jain VK, Chhabra DK. Choroid plexus papillomas of the cerebellopontine angle. Neurol India 2002; 50(3):352-358

9 Talacchi A, De Micheli E, Lombardo C, Turazzi S, Bricolo A. Choroid plexus papilloma of the cerebellopontine angle: a twelve patient series. Surg Neurol 1999;51(6):621-629

10 Tanaka K, Sasayama T, Nishihara M, et al. Rapid regrowth of an atypical choroid plexus papilloma located in the cerebellopontine angle. J Clin Neurosci 2009;16(1):121-124

11 Bonneville F, Savatovsky J, Chiras J. Imaging of cerebellopontine angle lesions: an update. Part 1: enhancing extra-axial lesions. Eur Radiol 2007;17(10):2472-2482

12 Casar Borota O, Jacobsen EA, Scheie D. Bilateral atypical choroid plexus papillomas in cerebellopontine angles mimicking neurofibromatosis 2. Acta Neuropathol 2006;111(5):500-502

13 Li S, Savolaine ER. Imaging of atypical choroid plexus papillomas. Clin Imaging 1996;20(2):85-90

14 Martin N, Pierot L, Sterkers O, Mompoint D, Nahum H. Primary choroid plexus papilloma of the cerebellopontine angle: MR imaging. Neuroradiology 1990;31(6):541-543

15 Tasdemiroglu E, Awh MH, Walsh JW. MRI of cerebellopontine angle choroid plexus papilloma. Neuroradiology 1996;38(1): $38-40$

16 Bonneville F, Savatovsky J, Chiras J. Imaging of cerebellopontine angle lesions: an update. Part 2: intra-axial lesions, skull base lesions that may invade the CPA region, and non-enhancing extraaxial lesions. Eur Radiol 2007;17(11):2908-2920

17 Rovit RL, Schechter MM, Chodroff P. Choroid plexus paillomas. Observations on radiographic diagnosis. Am J Roentgenol Radium Ther Nucl Med 1970;110(3):608-617

18 Peyre M, Bah A, Kalamarides M. Multifocal choroid plexus papillomas: case report. Acta Neurochir (Wien) 2012;154(2):295-299

19 Karim A, Fowler M, McLaren B, Cardenas R, Patwardhan R, Nanda A. Concomitant choroid plexus papillomas involving the third and fourth ventricles: a case report and review of the literature. Clin Neurol Neurosurg 2006;108(6):586-589

20 Krishnan S, Brown PD, Scheithauer BW, Ebersold MJ, Hammack JE, Buckner JC. Choroid plexus papillomas: a single institutional experience. J Neurooncol 2004;68(1):49-55

21 Picard C, Copty M, Lavoie G, Michaud J, Bouchard R. A primary choroid plexus papilloma of the cerebellopontine angle. Surg Neurol 1979;12(2):123-127

22 Sarkar C, Sharma MC, Gaikwad S, Sharma C, Singh VP. Choroid plexus papilloma: a clinicopathological study of 23 cases. Surg Neurol 1999;52(1):37-39

23 Tacconi L, Delfini R, Cantore G. Choroid plexus papillomas: consideration of a surgical series of 33 cases. Acta Neurochir (Wien) 1996;138(7):802-810
24 Jia DZ, Zhou MD, Jiang YQ Li G. Trigeminal neuralgia caused by a choroid plexus papilloma of the cerebellopontine angle: case report and review of the literature. J Int Med Res 2010;38(1): 289-292

25 Zhang W. Choroid plexus papilloma of the cerebellopontine angle, with special reference to vertebral angiographic study. Surg Neurol 1982;18(5):367-371

26 Doglietto F, Lauretti L, Tartaglione T, Gessi M, Fernandez E, Maira G. Diffuse craniospinal choroid plexus papilloma with involvement of both cerebellopontine angles. Neurology 2005;65(6):842

27 Zimny A, Sasiadek M. Contribution of perfusion-weighted magnetic resonance imaging in the differentiation of meningiomas and other extra-axial tumors: case reports and literature review. J Neurooncol 2011;103(3):777-783

28 Girardot C, Boukobza M, Lamoureux JP, et al. Choroid plexus papillomas of the posterior fossa in adults: MR imaging and gadolinium enhancement. Report of four cases and review of the literature. J Neuroradiol 1990;17(4):303-318

29 Jackson A, Panizza BJ, Hughes D, Reid H. Primary choroid plexus papilloma of the cerebellopontine angle: magnetic resonance imaging, computed tomographic and angiographic appearances. Br J Radiol 1992;65(777):754-757

30 García-Valtuille R, Abascal F, García-Valtuille AI, et al. Adult choroid plexus papilloma of the posterior fossa mimicking a hemangioblastoma. Case report. J Neurosurg 2000;92(5):870-872

31 Kieserman S, Linstrom C, McCormick S, Petschenik AJ. Choroid plexus papilloma of the cerebellopontine angle. Am J Otol 1996; 17(1):119-122

32 Cho YD, Choi GH, Lee SP, Kim JK. (1)H-MRS metabolic patterns for distinguishing between meningiomas and other brain tumors. Magn Reson Imaging 2003;21(6):663-672

33 Majós C, Cucurella G, Aguilera C, Coll S, Pons LC. Intraventricular meningiomas: MR imaging and MR spectroscopic findings in two cases. AJNR Am J Neuroradiol 1999;20(5):882-885

34 Krieger MD, Panigrahy A, McComb JG, et al. Differentiation of choroid plexus tumors by advanced magnetic resonance spectroscopy. Neurosurg Focus 2005;18(6A):E4

35 Horská A, Ulug AM, Melhem ER, et al. Proton magnetic resonance spectroscopy of choroid plexus tumors in children. J Magn Reson Imaging 2001;14(1):78-82

36 Shibata Y, Katayama W, Kawamura H, Anno I, Matsumura A. Proton magnetic resonance spectroscopy and 201Thallium-, 99mTechnetium methoxyisobutylisonitrile single photon emission computed tomography findings of a patient with choroid plexus papilloma. Neuroradiology 2008;50(8):741-742

37 Krishnan S, Brown PD, Scheithauer BW, Ebersold MJ, Hammack JE, Buckner JC. Choroid plexus papillomas: a single institutional experience. J Neurooncol 2004;68(1):49-55 